\title{
Bismuth and Erbium Co-doped Optical Fiber for a White Light Fiber Source
}

\author{
Dawei Song1, Jianzhong Zhang ${ }^{1,2^{*}}$, Shuo Fang ${ }^{1}$, Weimin Sun ${ }^{1}$, Zinat M. Sathi ${ }^{2}$, \\ Yanhuo Luo ${ }^{2}$, Gang-Ding Peng ${ }^{2}$ \\ ${ }^{1}$ Harbin Engineering University, Harbin 150001, China \\ ${ }^{2}$ School of Electrical Engineering \& Telecommunications, University of New South Wales, Sydney 2052, line NSW, Australia \\ Email: *zhangjianzhong@hrbeu.edu.cn
}

Received 2013

\begin{abstract}
We demonstrate a white light fiber source based on Bismuth and Erbium co-doped fiber and a single 830nm laser diode pump. The light spectral intensity from 1100 to $1570 \mathrm{~nm}$ is over $-45 \mathrm{dBm}$, which provide $\sim 40 \mathrm{~dB}$ dynamic range for an OSA based spectral measurement.
\end{abstract}

Keywords: Bi/Er Co-doped Fiber; White Light Sources; Optical Spectral Measurement

\section{Introduction}

Broadband fiber light sources and amplifiers have broad applications in the areas of optical communications, the spectral measurements of fiber devices and optical fiber sensor. Super-Lum-diodes and Er doped fiber based ASE sources are the main choices and however they are limited by the spectral bandwidth, normally smaller than $100 \mathrm{~nm}$. The super continue fiber light sources have the broadest spectrum, from $400 \mathrm{~nm}$ to $1700 \mathrm{~nm}$, and while its applications are limited by the comparably higher price especially for simple device measurement and sensing application. So the cheap and reliable fiber broadband sources need to be developed. Bi doped glass or silica material [1-5] show the broad luminescence and the low $\mathrm{Bi}(<0.02$ at \%) doped optical fiber[6-11] with low background loss has been developed to expend the new band window for optical communication and develop the high power laser. However, no $\mathrm{Bi}$ doped fiber based white light source is reported yet and it is difficult to be realized especially based on a single pump. Here we demonstrate a white light fiber source based our developed $\mathrm{Bi}$ and Er co-doped fiber [12] and a single cheap $830 \mathrm{~nm}$ laser diode pump. Here we achieved the stronger and applicable broadband light emission, and its spectral intensity is over $-45 \mathrm{dBm}$ from $1100 \mathrm{~nm}$ to $1570 \mathrm{~nm}$. The mechanism of the ultra-broadband source is discussed as well.

\section{Experiments and Discussions}

${ }^{*}$ Corresponding author.
The fiber is fabricated by in situ MCVD doping with concentrations of $[\mathrm{Er} 2 \mathrm{O} 3] \sim 0.01,[\mathrm{Al} 2 \mathrm{O} 3] \sim 0.15$, $[\mathrm{Bi} 2 \mathrm{O} 3] \sim 0.16,[\mathrm{P} 2 \mathrm{O} 5] \sim 0.94$, and $[\mathrm{GeO} 2] \sim 12.9 \mathrm{~mol}$ $\%$, respectively. The fiber has a numerical aperture NA 0.19 and core diameter of $3.2 \mu \mathrm{m}$ in order to achieve a cut-off wavelength $\lambda$ co $\sim 0.8 \mu \mathrm{m}$ because of our following pump laser diode of $830 \mathrm{~nm}$ in wavelength. We observe the backward luminescence spectra of our $\mathrm{Bi} / \mathrm{Er}$ co-doped fiber, pumped by an $830 \mathrm{~nm}$ laser diode with a maximum $60 \mathrm{mw}$ power, base on an 810/1310 $\mathrm{nm}$ WDM and an OSA (Agilent 86140B), shown in Figure 1. A power meter is used to monitor the pump power and the left pump power.

Broadband fiber light source: The strongest emission spectrum, based on the $60 \mathrm{mw}$ pump and $3.5 \mathrm{~m}$ long Bi/Er co-doped fiber, is shown in Figure 2(a). The red and green curves are corresponding to the measured and recovered true spectra, respectively. The true spectrum is the true emission spectrum that has been modulated by the $810 / 1310 \mathrm{~nm}$ WDM coupler. The true spectral intensity is over $-45 \mathrm{dBm}$ from $1100 \mathrm{~nm}$ to $1600 \mathrm{~nm}$ and over $-50 \mathrm{dBm}$ from $900 \mathrm{~nm}$ to $1100 \mathrm{~nm}$. It provides $\sim 40 \mathrm{~dB} \mathrm{dy}-$ namic measurement range, because of the OSA spectral measurement limitation of $\sim-85 \mathrm{dBm}$ at the resolution of $10 \mathrm{~nm}$, for the broadband spectral measurement of kinds

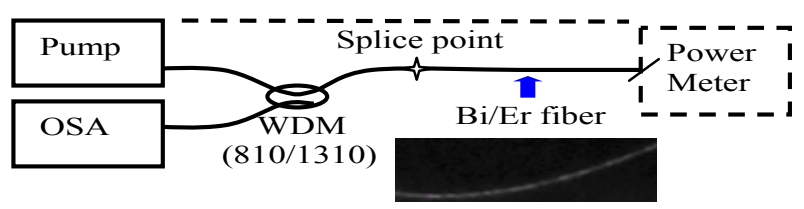

Figure 1. Experimental setup. 
of fiber devices. The broadband emission intensity is limited by the pump power by hands and it could be enhanced by using a pump with a higher power and a longer fiber. Figure 2(b) shows the relationship between the pump power and relative emission intensity of total spectrum at the range of $900 \mathrm{~nm}$ to $1600 \mathrm{~nm}$. Here the splice loss between lead-in single mode fiber and our Bi/Er co-doped fiber is around $\sim 1.5 \mathrm{~dB}$ because the mode field is not matched. We measured the spectra every $10 \mathrm{~min}$ and last for a few hours. The stand deviation $(<0.2 \mathrm{~dB})$ of the broadband spectrum in Figure 2(c) shows its good stability, which is important for the spectral measurement application.

Broadband emission observation: First, we measure the emission spectra excited by tunable laser with a wavelength range of $1259 \mathrm{~nm}-1500 \mathrm{~nm}$ (limited by our Agilent 8164B) and a constant power of $70 \mathrm{uW}$ and the results are shown in Figure 3(a). There are two obvious emission band and their central wavelengths are at $1420 \mathrm{~nm}$ and $1530 \mathrm{~nm}$, which come from $\mathrm{Bi}$ and Er related color centers. Second we observe the luminescence spectra of a section of our $\mathrm{Bi} / \mathrm{Er}$ co-doped fiber $(<10 \mathrm{~cm})$ based on $830 \mathrm{~nm}$ laser diode with different pump power, shown in Figures 3(b) and (d). The different increasing speeds of the spectra at different wavelength can be observed readily. We calculated the increased emission at the different wavelength areas when increasing the pump power from $0 \mathrm{~mW}$ to $1 \mathrm{mw}$, from $10 \mathrm{~mW}$ to $15 \mathrm{~mW}$, and from $55 \mathrm{mw}$ to $60 \mathrm{mw}$, shown in Figure 3(c). It is obvious that the emission band at $\sim 1420 \mathrm{~nm}$ is excited and saturated first and the left side emission bands from $900 \mathrm{~nm}-1200 \mathrm{~nm}$ come

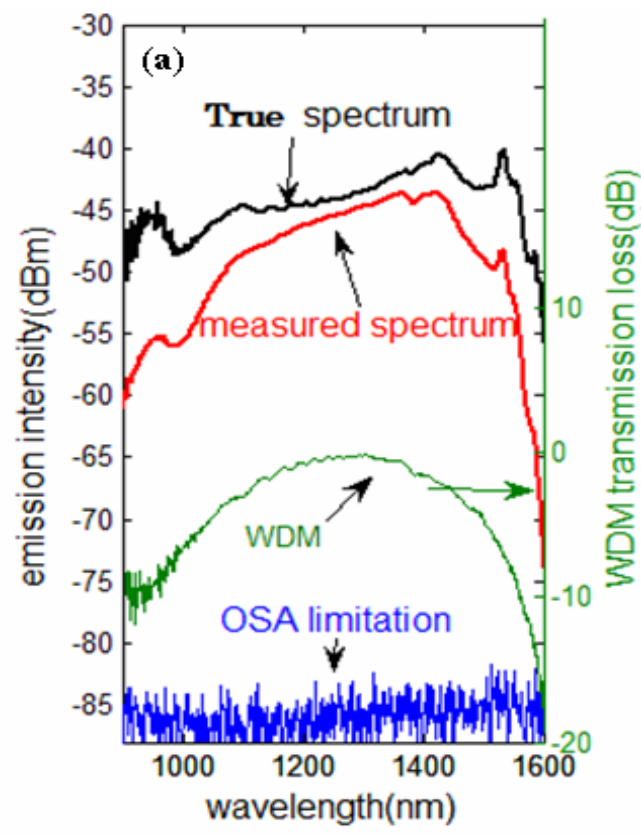

secondly and saturated later. The careful observation and analysis of such broadband light spectra is needed and done based on the more detail emission observation.

Broadband mechanism discussion: The Er irons give emission to support the $\mathrm{C}$ and $\mathrm{L}$ band as we expected. Its emission spectrum is still there because of its inter-shell structure according to emission observation. The Bi related Emission covers the band from $1000 \mathrm{~nm}$ to 1500 $\mathrm{nm}$. The emission of our $\mathrm{Bi} / \mathrm{Er}$ co-doped fiber demonstrated the emission around $1200 \mathrm{~nm}$ (Bi related) and 1530 $\mathrm{nm}$ (Er related) when pumped at $980 \mathrm{~nm}$, which is different from the case of $830 \mathrm{~nm}$ pump. We also observed the blue- green up-conversion emission, recorded by a camera and shown in Figure 1, when the pump power is over a few milliwatts, which also means the broader spectrum based on our system. We don't calibrate the power of this part of spectra because of the spectral limitation of our OSA. We believe that more than two kinds of $\mathrm{Bi}$ related color centers are involved in our $\mathrm{Bi} / \mathrm{Er}$ co-doped fiber. Bi-Si related color centers, reported in [9], correspond to the emission band around $1400 \mathrm{~nm}$, shown as the blue-line in Figure 3(c). We still find that the Bi related emission around $1200 \mathrm{~nm}$ is related to the Er concentration closely. The emission near $1200 \mathrm{~nm}$ would become lower when the Er concentration is lower for the $830 \mathrm{~nm}$ pump. It may be caused by the energy transition between $\mathrm{Er}$ and $\mathrm{Bi}$ and they maybe form a combined color centers. It is important to understand the broadband mechanism and improve the emission efficiency and the further detail experiments are processing, which expect to be reported at the conference.
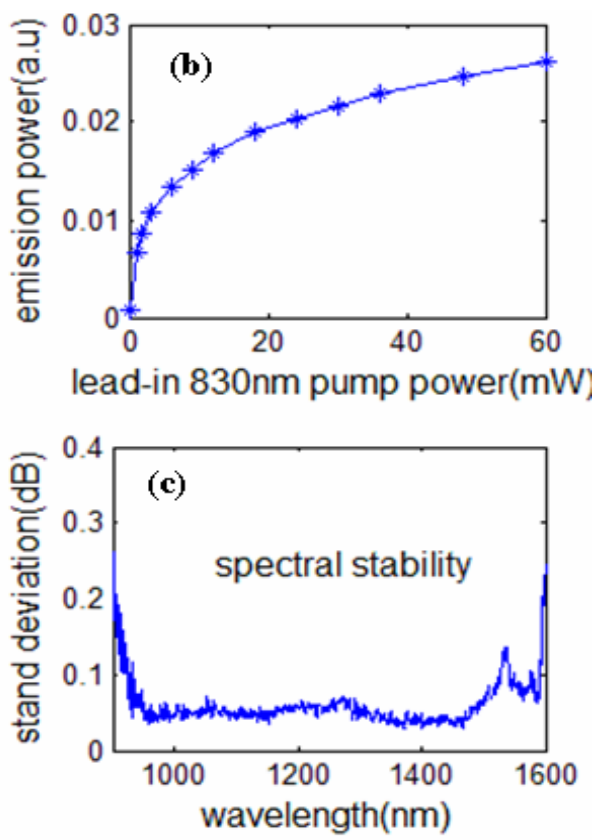

Figure 2. (a) Broadband emission, (b) the total emission power changed with the pump power, and (c) the standard deviation of the broadband spectrum. 

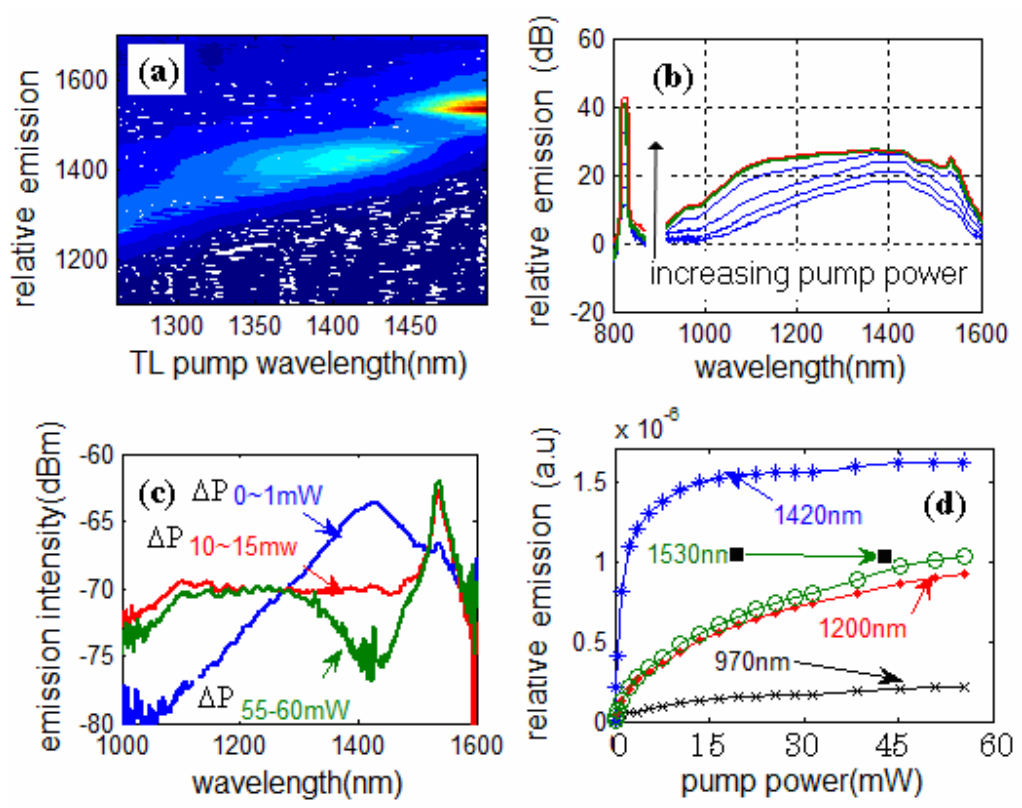

Figure 3. (a) Tunable laser based excitation-emission spectra, (b) the emission spectra of a short section of Bi Er co-doped fiber when increasing the 830nm pump power, (c) the increased emission between different pump powers, and (d) the relationship between the pump power and the emissions at different wavelengths.

\section{Discussion and Conclusions}

In conclusion, we report a fiber white light source from $1000 \mathrm{~nm}$ to $1570 \mathrm{~nm}$ based on the $\mathrm{Bi} / \mathrm{Er}$ co-doped fiber and a single $830 \mathrm{~nm}$ laser diode pump and discuss its mechanism based on emission observation, which expects to have applications in the spectral measurements of optical fiber devices and the optical fiber sensing.

\section{Acknowledgements}

Authors thank for the support by National Science foundation projects (60907034, 61077063, 11178010 and LBHZ10195), China Postdoctoral Science Foundation funded project (20100480965), Harbin Science foundation (2011 RFLXG004) and the Fundamental Research Funds of the Central University, China. Authors thank the support by international science linkages (ISL) project (CG130013) from the department of industry, Innovation, Science and Research (DIISR), Australia. An Australian Research council (ARC) LIEF grant helped to fund the national fiber facility at UNSW.

\section{REFERENCES}

[1] Y. Fujimoto and M. Nakatsuka, "Infrared luminescence from bismuth-doped silica glass," Japanese Journal of Applied Physics, Vol. 40, 2001, p.L279. doi:10.1143/JJAP.40.L279

[2] M. Y. Peng, J. R. Qiu, D. Chen, X. Meng and C. Zhu, "Broadband Infrared Luminescence from $\mathrm{Li}_{2} \mathrm{O}-\mathrm{Al}_{2} \mathrm{O}_{3}$ $\mathrm{ZnO}-\mathrm{SiO}_{2}$ Glasses Doped with $\mathrm{Bi}_{2} \mathrm{O}_{3}$," Optics Express, Vol.13, No. 18, 2005, pp. 6892-6898.

\section{doi:10.1364/OPEX.13.006892}

[3] Y. Kuwada, Y. Fujimoto and M. Nakatsuka, "Ultrawideband Light Emission from Bismuth and Erbium Doped Silica," Japanese Journal of Applied Physics, Vol.46, 2007, pp. 1531-1532. doi:10.1143/JJAP.46.1531

[4] J. Ren, G. Dong, S. Xu, R. Bao and J. Qiu, "Inhomogeneous Broadening, Luminescence Origin and Optical Amplification in Bismuth-Doped Glass," Journal of Chemical Physics, Vol.112, No.14, 2008, pp. 3036-3039. doi:10.1021/jp709987r

[5] M. Peng, N. Zhang, L. Wondraczek, J. Qiu, Z. Yang and Q. Zhang, "Ultrabroad NIR Luminescence and Energy Transfer in Bi and Er/Bi Co-Doped Germanate Glasses," Optics Express, Vol. 19, No. 21, 2011, pp. 20799-20807. doi:10.1364/OE.19.020799

[6] H. Y. Tam, W. H. Chung, B. O. Guan, H. L. Liu, P. K. A. Wai and N. Sugimoto, "Development of Bi2O3-Based Erbium-Doped Fibers," Proceedings of SPIE, Vol.5644, No.259,2005. doi:10.1117/12.577530

[7] V. V. Dvoyrin, V. M. Mashinsky, E. M. Dianov, A. A. Umnikov, M. V. Yashkov and A. N. Guranov, "Absorption, Fluorescence and Optical Amplification in MCVD Bismuth-Doped Silica Glass Optical Fibres," ECOC Proceeding, Vol.4, No. 949, 2005.

[8] I. A. Bufetov and E. M. Dianov, "Bi-Doped Fiber Lasers,"LaserPhysicsLetters,Vol.6,No.7,2009,pp.487-504. doi:10.1002/lapl.200910025

[9] S. V. Firstov et al. "Combined Excitation-Emission Spectroscopy of Bismuth Active Centers in Optical Fibers" Optics Express, Vol. 19, No. 20, 2011, pp. 19551-19561. doi:10.1364/OE.19.019551

[10] J. H. Shin and J. H. Lee, "Investigation of Signal Excited-State Absorption in Bismuth-Based Erbium-Doped Fiber Amplifier," Journal of the Optical Society of America, Vol. 27, No. 7, 2010, pp. 1452-1457. 
doi:10.1364/JOSAB.27.001452

[11] Y. Q. Qiu, X. Y. Dong and C. L. Zhao, "Spectral Characteristics of the Erbium-Bismuth Co-Doped Silica Fibers and its Application in Single Frequency Fiber Laser," Laser Physics, Vol. 20, No. 6, 2010, pp. 1418-1424. doi:10.1134/S1054660X10110186
[12] Y. H. Luo, J. X. Wen, J. Z. Zhang, J. Canning and G. D. Peng, "Bismuth and Erbium Codoped Optical Fiber with Ultra Broadband Luminescence Across O-, E-, S-, C-, and L-bands" Optics Letters, Vol. 37, No. 16, 2012, pp. 3447-3449.

doi:10.1364/OL.37.003447 